\title{
Eficácia de intervenções educacionais sobre os conhecimentos em nutrição e atividade física de escolares
}

\section{Effectiveness of educational interventions on the knowledge in nutrition and physical activity in schoolchildren}

Efectividad de las intervenciones educativas en el conocimiento de la nutrición y actividad física de los niños en edad escolar

Liana Marczal ${ }^{1}$ Luana Bernardi ${ }^{2}$ Daiana Novello ${ }^{3}$

${ }^{1}$ Graduanda em Nutrição pela Universidade Estadual do Centro-Oeste (UNICENTRO), Campus de Cedeteg, Guarapuava, Paraná, Brasil. E-mail: lianamarczal@gmail.com

${ }^{2}$ Nutricionista, Mestre. Docente do Curso de Nutrição da Universidade Estadual do Centro-Oeste (UNICENTRO), Campus de Cedeteg, Guarapuava, Paraná, Brasil. E-mail: luana_bernardi@yahoo.com.br

${ }^{3}$ Nutricionista, Doutora. Docente do Curso de Nutrição e do Mestrado Interdisciplinar em Desenvolvimento Comunitário da Universidade Estadual do Centro-Oeste (UNICENTRO), Campus de Cedeteg, Guarapuava, Paraná, Brasil. E-mail: nutridai@gmail.com 
Resumo: Objetivou-se avaliar a eficácia de intervenções educacionais interdisciplinares sobre os conhecimentos em nutrição e atividade física de escolares. Participaram da pesquisa 458 crianças de 7 a 10 anos, matriculadas em 16 escolas municipais. Os conhecimentos sobre nutrição e atividade física foram avaliados por meio de questionários aplicados antes e após as intervenções, as quais foram realizadas por 5 meses, sendo dois encontros/mês, totalizando 10 encontros/escola. Em relação aos conhecimentos em nutrição e atividade física pré-intervenção, verificou-se, respectivamente, que 15,7\% e $12,9 \%$ das crianças tinham baixos conhecimentos, $45,2 \%$ e $44,1 \%$ bons conhecimentos e; $39,1 \%$ e 43,0\% ótimos conhecimentos. No período pós-intervenção, 0,2\% e 0,2\% das crianças demonstraram baixos conhecimentos; $1,3 \%$ e 2,4\% bons conhecimentos e; $98,5 \%$ e $97,4 \%$ ótimos conhecimentos. As intervenções educativas realizadas no ambiente escolar favorecem o aprendizado das crianças, promovendo um consumo alimentar consciente e hábitos mais saudáveis.

Palavras-chave: ações em saúde; interdisciplinaridade; promoção da saúde.

Abstract: This study aimed to evaluate the effectiveness of educational interventions on interdisciplinary knowledge in nutrition and physical activity in school. The participants were 458 children aged 7 to 10 years, enrolled in 16 public schools. Knowledge about nutrition and physical activity were assessed using questionnaires before and after the interventions, which were carried out for 5 months, 2 meetings/month, totaling 10 meetings/school. Regarding knowledge on nutrition and physical activity before the intervention, there was, respectively, $15.7 \%$ and $12.9 \%$ of children had low knowledge, $45.2 \%$ and $44.1 \%$ good knowledge and; $39.1 \%$ and $43.0 \%$ great knowledge. In the postintervention period, $0.2 \%$ and $0.2 \%$ of the children showed low knowledge; $1.3 \%$ and $2.4 \%$ good knowledge and; $98.5 \%$ and $97.4 \%$ great knowledge. Educational interventions in the school environment promote children's learning, promoting a conscious food consumption and healthier habits.

Key words: health actions; interdiciplinarity; health promotion.

Resumen: Este estudio tuvo como objetivo evaluar la efectividad de las intervenciones educativas interdisciplinarios en el conocimiento en la nutrición y la actividad física de los estudiantes. Los participantes fueron 458 niños de 7 a 10 años, matriculados en 16 escuelas municipales. El conocimiento sobre la nutrición y la actividad física se evaluó mediante cuestionarios antes y después de las intervenciones, que se llevaron a cabo durante 5 meses, 2 encuentros/mes, por un total de 10 encuentros/escuela. Con respecto al conocimiento en la nutrición y la actividad física antes de la intervención, se encontró, respectivamente, que $15,7 \%$ y el $12,9 \%$ de los niños tenían conocimientos bajos, $45,2 \%$ y $44,1 \%$ buenos conocimientos; y $39,1 \%$ y $43,0 \%$ gran conocimientos. En el período posterior a la intervención, $0,2 \%$ y $0,2 \%$ de los niños mostraron bajos conocimientos; $1,3 \%$ y el $2,4 \%$ buenos conocimientos; y $98,5 \%$ y $97,4 \%$ gran conocimientos Las intervenciones educativas en el entorno escolar favorecen el aprendizaje de los niños, promoviendo un consumo alimentar consciente y hábitos más saludables.

Palabras clave: acciones de salud; interdisciplinariedad; promoción de la salud. 


\section{INTRODUÇÃO}

A obesidade é conceituada como um aumento de tecido adiposo e peso corporal, que geralmente envolve fatores ambientais e genéticos (SIMON et al., 2009). Atualmente, verifica-se uma prevalência de excesso de peso infantil variando entre 32 a 40\% no Sudeste, Sul e Centro-Oeste, e de 25 a 30\% no Norte e Nordeste do Brasil (IBGE, 2010; FRIEDRICH et al., 2012). Nesse contexto, entre os anos de 2008 e 2009, houve um aumento no número de indivíduos obesos no país, ultrapassando os de desnutridos, caracterizando o fenômeno de transição nutricional, o qual ocorre mundialmente (FRIEDRICH et al., 2012). Esse fato resulta, especialmente, da redução na prática de atividade física e do elevado consumo de produtos com alto teor de calorias, carboidratos refinados, sal, açúcares e gorduras e baixo conteúdo de fibras, água, frutas e verduras (PONTES et al., 2009).

Apesar de o tipo de alimentação influenciar no prognóstico da obesidade infantil, deve-se considerar também os fatores psicológicos, biológicos, sociais, econômicos e comportamentais, os quais podem colaborar diretamente no estado nutricional futuro. Nesse caso específico, os distúrbios de comportamento alimentar e na relação familiar, principalmente em períodos de crescimento, podem acelerar o processo da obesidade (SIQUEIRA et al., 2009). Isso, porque os hábitos alimentares são formados ainda na infância. Outros aspectos também devem ser considerados quando há alteração no peso corporal, como o ambiente da refeição, meios de comunicação e tendências sociais, que da mesma forma associam-se às mudanças na dieta (OLIVEIRA, N. et al., 2010). As crianças também passam um grande período do dia na escola, o qual é determinante na formação e consolidação dos hábitos alimentares e práticas de atividades físicas adequadas, influenciando, inclusive, na redução do risco de patologias na idade adulta. Assim, o ambiente escolar pode ser considerado uma dimensão única 
de novos aprendizados e intervenções em saúde, considerando-se as características individuais, sociais, econômicas e regionais na qual a criança está inserida (FARIAS et al., 2010).

A prática de atividade física promove habilidades motoras e a capacidade de aperfeiçoar o desempenho, melhorando a autoestima dos indivíduos. Também auxilia na prevenção de doenças crônicas que podem se instalar desde a infância, como a diabetes mellitus, hipertensão arterial e, consequentemente, a obesidade (OLIVEIRA, J. et al., 2010). Nesse aspecto, ações que promovam a correção de hábitos alimentares, bem como a inserção de atividades físicas cotidianas para o público infantil devem ser consideradas como estratégias de saúde pública.

A promoção da saúde é uma importante forma de intervenção para fortalecer o processo saúde-doença-cuidado. Suas práticas conjuntas visam desenvolver conhecimentos e habilidades para o autocuidado em saúde e a prevenção das condutas de risco. No contexto educacional infantil, ações voltadas à alimentação saudável e prática de atividade física ganham grande destaque, pois, em geral, consideram aspectos locais e regionais para sua implementação. Citase como exemplo, os valores, condutas, condições sociais, culturais, ambientais e estilo de vida (YOKOTA et al., 2010), fatores esses que são analisados e utilizados para a confecção dos instrumentos de educação nutricional e práticas de atividade física, voltados ao contexto habitual da criança.

Para que a promoção da saúde possa ser eficaz, considera-se que, indispensavelmente, a equipe de trabalho deve atuar de forma multi e interdisciplinar. Também, no contexto escolar, as diversas áreas do conhecimento necessitam convergir para um bem comum, fato que vem demonstrando efeitos positivos na prevenção da obesidade e melhora da qualidade de vida (FREITAS et al., 2009). Dessa forma, ações conjuntas na escola são fundamentais para que se obtenha um 
aprendizado duradouro, inclusive em fases mais avançadas da vida como a adolescência e idade adulta, favorecendo um efeito preventivo e não curativo (FREITAS, 2009). Destaca-se que as intervenções precoces, realizadas ainda na infância, são mais eficientes que aquelas aplicadas na idade adulta (BERTIN et al., 2010).

O objetivo do estudo foi avaliar a eficácia de intervenções educacionais interdisciplinares sobre os conhecimentos em nutrição e atividade física de escolares.

\section{MATERIAL E MÉTODOS}

\subsection{População alvo}

Participaram da pesquisa crianças com idade entre 7 e 10 anos, matriculadas entre os 2 ㅇ e 5ㅇa anos de escolas públicas municipais da cidade de Guarapuava, PR.

O processo de amostragem foi realizado em múltiplas fases: a) listagem de todas as escolas de ensino fundamental da zona urbana da cidade de Guarapuava, PR (36); b) estratificação das escolas de acordo com os bairros da cidade (16); c) escolha de uma escola por bairro, priorizando aquela com maior representatividade de alunos; d) listagem de todas as turmas do 2으 a 5 ㅇan ano das escolas selecionadas (128) e; e) seleção da amostra de alunos de cada escola por meio do processo de amostragem aleatória simples. Para o cálculo amostral, foi considerada a prevalência média estimada de obesidade no país (50\%) (MELLO et al., 2010), com precisão de 5\%. Dessa forma, foram selecionadas 16 escolas, totalizando 458 crianças avaliadas.

\subsection{Atividades educativas}

As atividades educativas foram aplicadas por profissionais e acadêmicos da área de nutrição e de educação física. As interven- 
ções foram realizadas por 5 meses, sendo dois2 encontros por mês, totalizando 10 encontros por escola. Cada intervenção teve duração total de 30 minutos. Os primeiros 15 minutos foram utilizados para explicação dos temas, em seguida foram realizadas atividades expositivas e participativas. Os exercícios foram trabalhados em encontros intercalados e individuais, respeitando-se cada área de conhecimento. Destaca-se que, após a explicação teórica, foi aplicada uma dinâmica prática, reforçando o conhecimento teórico.

Em geral, as atividades possibilitaram aos alunos uma identificação e reflexão de problemas, levantamento de hipóteses e apontamento de soluções para reduzir o sedentarismo. Para isso, foram incluídas palestras educativas e dinâmicas, por meio de uma linguagem simples, permitindo a fixação e compreensão do conteúdo pela faixa etária estudada. Durante todo o processo educacional, foi priorizada a participação ativa e a interação dos escolares.

As atividades lúdico-educativas referentes à nutrição foram elaboradas segundo o Guia da Pirâmide Alimentar (BRASIL, 2006; PHILIPPI, 2014), que reflete visualmente conceitos alimentares como variedade, proporção e moderação. Os temas abordados foram: grupos alimentares; alimentos e suas diferentes funções no organismo; e porções diárias recomendadas (PHILIPPI, 2014). Essa ação teve o objetivo de construir, com o auxílio das crianças, os níveis da pirâmide a partir de explicações prévias, promovendo um menor consumo de alimentos calóricos e maior ingestão de alimentos com elevado teor de nutrientes, estimulando uma alimentação saudável e variada. Todas as ações relacionadas à nutrição foram realizadas nas salas de aula e no pátio da escola, o que facilitou o aprendizado.

As temáticas relacionadas à atividade física tiveram como base o conteúdo abordado em diretrizes (BRASIL, 2008; DHHS, 2008), manuais (SBP, 2008) e revistas para crianças de 6 a 9 anos (PHAC, 2002). Os materiais contemplavam recomendações de atividade física para 
crianças e adolescentes, abrangendo informações sobre a quantidade, tipos e intensidade; benefícios à saúde; e incentivo para a prática diária de atividades e brincadeiras. Além disso, foram contemplados temas relacionados aos problemas ocasionados pela inatividade física; o sedentarismo na infância, enfatizando, principalmente, o uso prolongado de aparelhos eletrônicos (ex. televisão, computador e videogame); e estímulo à prática de brincadeiras que resgatavam a cultura popular e que exigiam movimento corporal, como caminhar, correr e pular. O objetivo da ação foi conscientizar a criança para a prática de exercícios diários, tanto na escola quanto externamente, além de promover atividades interativas entre os grupos. As intervenções foram realizadas em um local próprio da escola como, por exemplo, a quadra de educação física.

Na Tabela 1 estão descritas as atividades relacionadas à nutrição e à atividade física realizadas nas escolas.

Tabela 1 - Atividades de educação nutricional e de atividade física desenvolvidas nas escolas de Guarapuava, PR, Brasil, 2015

\section{Mês Encontro Atividade}

Integração dos participantes: apresentação individual e descrição

1A das preferências alimentares e de atividades físicas. Aplicação dos questionários sobre nutrição e atividade física. Explicação sobre o grupo dos pães, cereais, raízes e tubérculos.

$1 B$ Desenvolvimento da dinâmica "Caça aos carboidratos" (PRADO, 2011).

Explicação sobre "Os benefícios da atividade física para a saúde".

2A Aplicação da atividade "Caça palavras", com o intuito de encontrar as palavras-chave que foram abordadas durante a explicação.

2 Explicação sobre os grupos das hortaliças e frutas.

2B Desenvolvimento da dinâmica "O Baú da alimentação saudável" (BERNART; ZANARDO, 2011). 


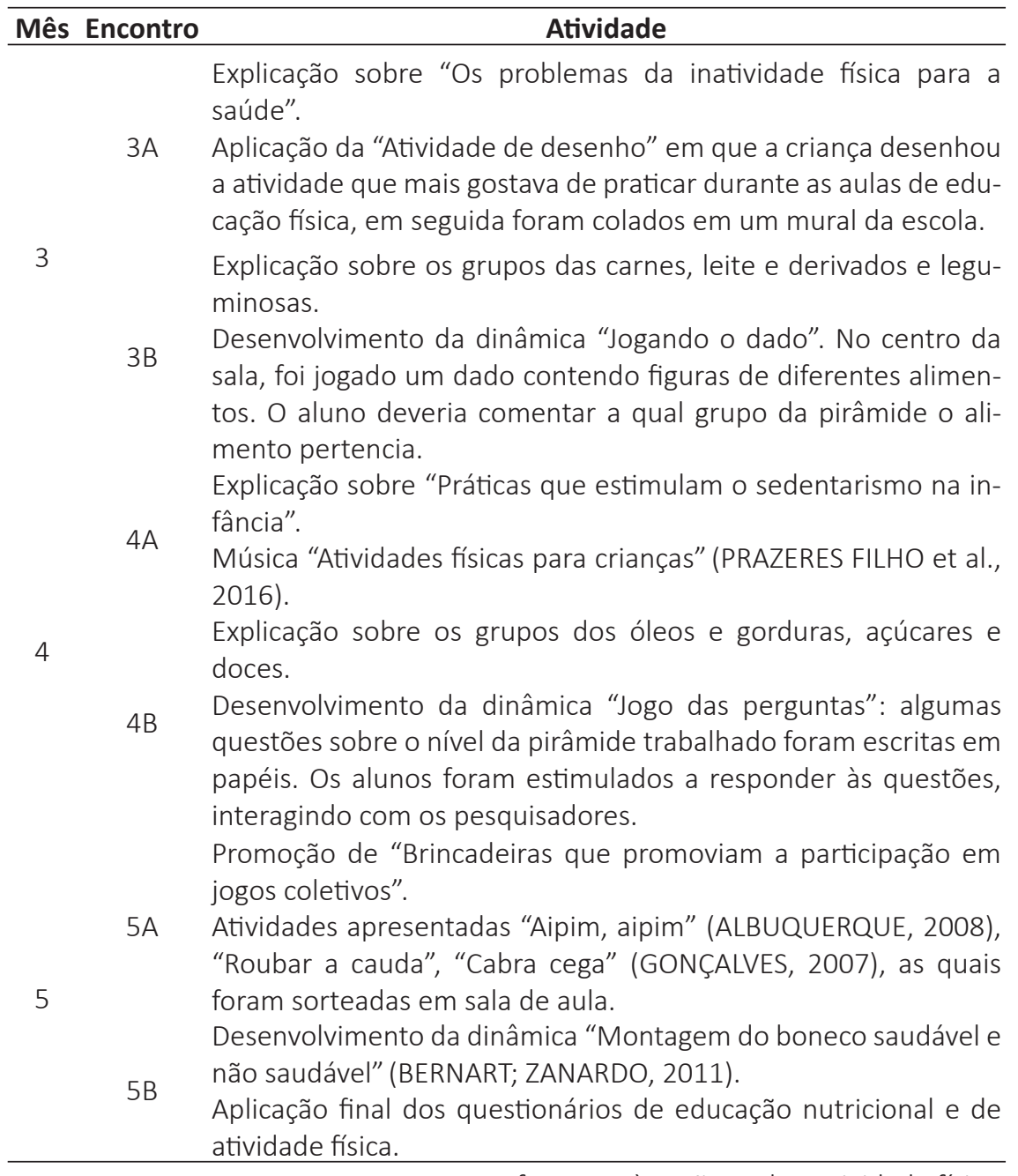

Nota: Os encontros 1A, 2A, 3A, 4A e $5 A$ referem-se às ações sobre atividade física; os encontros 1B, 2B, 3B, 4B e 5B referem-se às ações sobre nutrição; total de encontros por escola $=10$.

\subsection{Avaliação das atividades educativas}

Como forma de avaliação do programa intervencionista, os participantes responderam a dois instrumentos: um sobre conhecimentos 
em nutrição e outro sobre conhecimentos em atividade física, que foram aplicados em dois momentos: a) no primeiro encontro, antes da realização das ações educativas (pré-intervenção) e; b) no último encontro, após todas as ações educativas (pós-intervenção).

A elaboração do questionário sobre nutrição baseou-se no conteúdo apresentado no Guia da Pirâmide Alimentar (PHILIPPI, 2014). Dessa forma, tinha como objetivo verificar o conhecimento acerca dos grupos alimentares- alimentos fontes de carboidratos, vitaminas e minerais, proteínas, açúcares e lipídios, pertencentes em cada grupo da pirâmide alimentar.

O instrumento sobre atividade física foi desenvolvido com base nos questionários de Domingues et al. (2004) e Silveira e Silva (2011). Sua finalidade foi investigar o entendimento das crianças referente aos benefícios da prática de atividade física para a saúde, prevenção de doenças crônicas e seus efeitos no organismo humano.

Os questionários (conhecimentos nutrição e conhecimentos atividade física) eram compostos por quatro questões gerais ilustradas de múltipla escolha. Cada pergunta apresentava oito alternativas, sendo apenas quatro corretas. Cada pergunta tinha peso um, sendo que o escore final variava de zero a quatro pontos por questão. A pontuação variou entre zero e 16 pontos. A classificação dos conhecimentos em nutrição e atividade física baseou-se em estudo de Bertin et al. (2010), adaptando-se a pontuação da seguinte forma: "baixos conhecimentos" (zero a cinco pontos), "bons conhecimentos" (seis a 10 pontos) e, "ótimos conhecimentos" (11 a 16 pontos).

\subsection{Análise de dados}

Os dados foram analisados com auxílio do programa estatístico Statistical Package for Social Sciences (SPSS ${ }^{\oplus}$ versão 20). A amostra foi caracterizada inicialmente pela análise descritiva. Para a avaliação do 
conhecimento sobre nutrição e atividade física pré e pós-intervenção foram aplicados os testes de Qui-quadrado, Fisher, MacNemar e t de student pareado, todos com um nível de significância de $5 \%(p<0,05)$.

\subsection{Questões éticas}

O projeto foi aprovado pelo Comitê de Ética em Pesquisa Envolvendo Seres Humanos (COMEP) da UNICENTRO, sob parecer $\mathrm{n}$. 1.064.507/2015.

Os critérios de inclusão para seleção dos alunos foram: ter idade entre 7 a 10 anos; estar matriculado regularmente na escola (2은 e 50 ano); participar em todas as atividades propostas na pesquisa; e assinar o Termo de Assentimento (TA) e apresentar o Termo de Consentimento Livre e Esclarecido (TCLE) assinado pelo responsável legal. As crianças foram excluídas quando não atendiam aos critérios de inclusão.

\section{RESULTADOS E DISCUSSÃO}

Foram avaliadas 458 crianças ( 2 ㅇ e 5ㅇano) submetidas à intervenção sobre nutrição e atividade física. A faixa etária foi de 7 a 10 anos $(8,11 \pm 0,74$ anos), e 48,9\% ( $n=224)$ eram do sexo masculino e $51,1 \%(n=234)$ do sexo feminino.

Em relação aos temas avaliados nos questionários sobre nutrição e atividade física pré-intervenção, foi verificado que as crianças apresentavam, respectivamente: 39,1\% (179) e 43,0\% (197) ótimos conhecimentos; 45,2\% (207) e 44,1\% (202) bons conhecimentos e; $15,7 \%$ (72) e 12,9\% (59) baixos conhecimentos. Após a intervenção, observou-se uma melhora geral no entendimento das crianças quanto aos aspectos nutricionais e de atividade física, respectivamente: 98,5\% (451) e 97,4\% (446) ótimos conhecimentos; 1,3\% (6) e 2,4\% (11) bons conhecimentos e; 0,2\% (1) e 0,2\% (1) baixos conhecimentos. 
Os resultados demonstram uma melhora considerável nos conhecimentos das crianças em ambos os temas avaliados após as atividades intervencionistas, sendo que quase $100 \%$ das crianças apresentaram ótimos conhecimentos. Efeitos similares foram verificados por Oliveira et al. (2011), que avaliaram o nível de conhecimento de crianças (7 a 11 anos) em relação à nutrição, antes e após a utilização de teatro de fantoches. De forma semelhante, Silva e Costa (2011) observaram um aumento no conhecimento sobre prevenção de doenças crônicas e intenção de mudanças de hábitos alimentares e prática de atividade física entre crianças e adolescentes, após aplicação de intervenções educativas na área.

Segundo Coscrato et al. (2010), ações educativas em escolas têm uma influência direta nas mudanças comportamentais de crianças. Esse efeito ocorre porque, geralmente, são utilizadas formas lúdicas de aprendizado, o que aumenta o interesse no assunto, fato confirmado no presente trabalho. Cabe destacar que a avaliação é fundamental no processo de aprendizagem, uma vez que permite realizar uma análise das ações realizadas, verificando sua eficácia e, assim, possibilitando reformulações e melhorias nas ações (YOKOTA et al., 2010).

A Tabela 2 apresenta a prevalência de acertos para as ações de nutrição e de atividade física pré e pós-intervenção. 
Tabela 2 - Prevalência de acertos das crianças para os níveis de conhecimento (baixo e bom/ótimo), em relação aos temas de nutrição e de atividade física, pré e pós-intervenção, Guarapuava, PR, Brasil, 2015

\begin{tabular}{|c|c|c|c|c|c|c|}
\hline \multirow{3}{*}{ Conhecimentos } & & \multicolumn{4}{|c|}{ Pós-intervenção } & \multirow{3}{*}{$\mathbf{p}^{\mathrm{a}}$} \\
\hline & & \multicolumn{2}{|c|}{ Baixo } & \multicolumn{2}{|c|}{ Bom/ótimo } & \\
\hline & & $(\%)$ & $\mathrm{n}$ & (\%) & $\mathrm{n}$ & \\
\hline Nutrição & & & & & & 0,000 \\
\hline \multirow{2}{*}{ Pré-intervenção } & Baixo & 0,0 & 0 & 15,7 & 72 & \\
\hline & Bom/ótimo & 0,2 & 1 & 84,1 & 385 & \\
\hline Atividade física & & & & & & 0,000 \\
\hline \multirow{2}{*}{ Pré-intervenção } & Baixo & 0,0 & 0 & 12,9 & 59 & \\
\hline & Bom/ótimo & 0,2 & 1 & 86,9 & 398 & \\
\hline
\end{tabular}

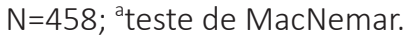

Constatou-se que 15,7 e 12,9\% das crianças que tinham baixos conhecimentos em nutrição e atividade física respectivamente no período pré-intervenção, passaram a apresentar bons/ ótimos conhecimentos após as intervenções $(p<0,05)$ (Tabela 2). Dessa forma, conclui-se que as técnicas utilizadas foram altamente eficazes para a aprendizagem dos alunos. Esses dados corroboram com Oliveira et al. (2011), que verificaram um aumento de crianças que apresentavam ótimos conhecimentos em nutrição $(67,83 \%)$ após o período de intervenção.

Diante das avaliações da presente pesquisa, verifica-se a necessidade contínua de ações em saúde no ambiente escolar. Esse fato que pode colaborar para a melhoria no estado nutricional do público infantil, uma vez que crianças obesas apresentam, em geral, menores conhecimentos sobre sua saúde (BERTIN et al., 2010). Carmo et al. (2013) ressaltam a importância do incentivo à prática de atividade física no ambiente escolar, como realizado na presente pesquisa. 
Isso, porque é nesta fase que os hábitos e os comportamentos de saúde são formados, refletindo-se na idade adulta. Nesse aspecto, pesquisas apontam que a atividade física regular pode reduzir o risco de ocorrência de doenças crônicas não transmissíveis, bem como meIhorar aspectos referentes ao aumento da força, resistência muscular, cardiorrespiratória e de flexibilidade e composição corporal (ENES; SLATER, 2010).

Apesar de as crianças demonstrarem bons conhecimentos sobre a importância da atividade física, atualmente verifica-se um alto nível de sedentarismo nessa fase. Alguns fatores podem explicar esse fato, tais como: envolvimento excessivo em tarefas intelectuais; baixa participação nas aulas de educação física; e utilização de equipamentos eletrônicos por longos períodos de tempo (televisão, videogame, computador, celular, dentre outros). Essas atividades podem reduzir as atividades realizadas ao ar livre e com maior gasto energético, colaborando, dessa forma, para o aumento da obesidade infantil (SILVA et al., 2009).

Na Tabela 3 está descrita a razão de chances (odds ratio) de acertos entre o sexo masculino e feminino, em relação aos temas de nutrição e de atividade física, pré e pós-intervenção. 
Tabela 3 - Análise bivariada e prevalência de acertos das crianças (sexo masculino e feminino) para os níveis de conhecimento (baixo e bom/ ótimo), em relação aos temas de nutrição e de atividade física, pré e pós-intervenção, Guarapuava, PR, Brasil, 2015

\begin{tabular}{|c|c|c|c|c|c|c|c|c|}
\hline \multirow[t]{2}{*}{ Conhecimentos } & & \multicolumn{2}{|c|}{$\begin{array}{c}\text { Sexo } \\
\text { masculino }\end{array}$} & \multicolumn{2}{|c|}{$\begin{array}{c}\text { Sexo } \\
\text { feminino }\end{array}$} & \multirow[t]{2}{*}{$\begin{array}{l}\text { Odds } \\
\text { ratio } \\
\text { bruto }\end{array}$} & \multirow[t]{2}{*}{$\begin{array}{c}\text { Intervalo de } \\
\text { confiança } \\
95 \%\end{array}$} & \multirow[t]{2}{*}{$\mathbf{p}^{\mathrm{a}}$} \\
\hline & & $\%$ & $n$ & $\%$ & $\mathrm{n}$ & & & \\
\hline \multicolumn{9}{|l|}{ Nutrição } \\
\hline \multirow[b]{2}{*}{ Pré-intervenção } & Baixo & 17,0 & 38 & 14,5 & 34 & 1,202 & $0,73-1,99$ & $0,474^{a}$ \\
\hline & $\begin{array}{l}\text { Bom/ } \\
\text { ótimo }\end{array}$ & 83,0 & 186 & 85,5 & 200 & & 1 & \\
\hline \multirow[b]{2}{*}{ Pós-intervenção } & Baixo & 0,4 & 1 & 0,0 & 0 & 2,049 & $1,87-2,25$ & $0,489^{b}$ \\
\hline & $\begin{array}{l}\text { Bom/ } \\
\text { ótimo }\end{array}$ & 99,6 & 223 & 100 & 234 & & 1 & \\
\hline \multicolumn{9}{|l|}{ Atividade física } \\
\hline \multirow[b]{2}{*}{ Pré-intervenção } & Baixo & 14,7 & 33 & 11,1 & 26 & 1,382 & $0,80-2,40$ & $0,248^{a}$ \\
\hline & $\begin{array}{l}\text { Bom/ } \\
\text { ótimo }\end{array}$ & 85,3 & 191 & 88,9 & 208 & & 1 & \\
\hline \multirow[b]{2}{*}{ Pós-intervenção } & Baixo & 0,0 & 0 & 0,4 & 1 & 1,961 & $1,79-2,15$ & $1,000^{b}$ \\
\hline & $\begin{array}{l}\text { Bom/ } \\
\text { ótimo }\end{array}$ & 100,0 & 224 & 99,6 & 233 & & 1 & \\
\hline
\end{tabular}

${ }^{\mathrm{a}}$ Teste de Qui-quadrado; ${ }^{\mathrm{b}}$ Teste exato de Fisher; $\mathrm{N}=458$.

Não houve diferença significativa $(p>0,05)$ entre os sexos referente ao número de acertos nos períodos pré e pós-intervenção, concordando com Silveira e Silva (2011). Geralmente, os conhecimentos das crianças são assimilados de forma espontânea, a partir de experiências diretas. Entretanto, no ambiente escolar, existe a preocupação de se desenvolver situações que propiciem o aprimoramento da capacidade intelectual, o que gera transformações constantes de pensamento. Assim, todas as crianças são expostas ao mesmo conteúdo de aprendizado, independente do gênero, proporcionando conhecimentos similares. Além disso, o envolvimento com o movimento corporal é muito grande em ambos os gêneros, elevando a participação em aulas de educação física. Essas in- 
Eficácia de intervenções educacionais sobre os conhecimentos em nutrição e atividade física de escolares

terações demonstram uma igualdade na forma de aprendizagem nessa fase da vida (FERREIRA; BARREARA, 2010), o que explica a semelhança na prevalência de acertos entre os gêneros (Tabela 3).

Na Tabela 4, observa-se a média e o percentual de acertos das crianças, em relação aos temas de nutrição e atividade física, pré e pós-intervenção.

Tabela 4 - Percentual e média de acertos das crianças em relação aos temas avaliados nos questionários sobre nutrição e atividade física, pré e pós-intervenção, Guarapuava, PR, Brasil, 2015

\begin{tabular}{|c|c|c|c|c|}
\hline \multirow[t]{2}{*}{ Temas avaliados } & \multicolumn{2}{|c|}{ Pré-intervenção } & \multicolumn{2}{|c|}{ Pós-intervenção } \\
\hline & $\%$ & média $\pm \mathrm{DP}$ & $\%$ & média $\pm D P$ \\
\hline \multicolumn{5}{|l|}{ Nutrição } \\
\hline Alimentos fonte de carboidratos & 38,48 & $1,54 \pm 1,18^{b}$ & 86,30 & $3,46 \pm 0,79^{a}$ \\
\hline $\begin{array}{l}\text { Alimentos fonte de vitaminas e min- } \\
\text { erais }\end{array}$ & 74,85 & $3,00 \pm 1,26^{b}$ & 92,30 & $3,69 \pm 0,62^{a}$ \\
\hline Alimentos fonte de proteínas & 45,96 & $1,84 \pm 1,16^{b}$ & 86,36 & $3,46 \pm 0,72^{a}$ \\
\hline $\begin{array}{l}\text { Alimentos fonte de açúcares e/ou } \\
\text { gordura }\end{array}$ & 80,46 & $3,22 \pm 1,08^{b}$ & 95,21 & $3,82 \pm 0,49^{a}$ \\
\hline Total de acertos & 59,74 & $9,56 \pm 3,47^{b}$ & 89,97 & $14,39 \pm 1,57^{\mathrm{a}}$ \\
\hline \multicolumn{5}{|l|}{ Atividade Física } \\
\hline $\begin{array}{l}\text { Doenças que podem ser evitadas pela } \\
\text { atividade física diária }\end{array}$ & 46,29 & $1,85 \pm 1,20^{b}$ & 84,77 & $3,39 \pm 0,87^{a}$ \\
\hline O que causa a falta de exercícios & 50,00 & $2,00 \pm 1,15^{b}$ & 84,56 & $3,39 \pm 0,86^{a}$ \\
\hline $\begin{array}{l}\text { O que melhora com a prática de ativi- } \\
\text { dade física }\end{array}$ & 71,67 & $2,87 \pm 2,27^{b}$ & 91,22 & $3,66 \pm 0,66^{a}$ \\
\hline $\begin{array}{l}\text { Quais atividades ajudam a ter uma } \\
\text { boa saúde }\end{array}$ & 80,57 & $3,22 \pm 1,10^{b}$ & 96,36 & $3,87 \pm 0,44^{a}$ \\
\hline Total de acertos & 61,33 & $9,81 \pm 3,51^{b}$ & 89,53 & $14,33 \pm 1,77^{\mathrm{a}}$ \\
\hline
\end{tabular}

Total de respostas corretas dos questionários: 16; DP: desvio padrão da média; Letras diferentes na linha indicam diferença significativa pelo teste t de student para amostras pareadas $(\mathrm{p}<0,05) ; \mathrm{N}=458$. 
Maiores percentuais e médias de acertos $(p<0,05)$, para todos os temas avaliados, foram constados no período pós-intervenção. Esse fato demonstra que as ações educativas proporcionaram um aumento do conhecimento sobre os assuntos entre o público infantil, corroborando com Oliveira et al. (2011), que avaliaram a aprendizagem de crianças sobre alimentação saudável.

Segundo Silva e Costa (2011), a atividade física desempenha papel fundamental sobre a condição física, psicológica e mental dos indivíduos. Também na fase escolar, a nutrição apresenta grande importância, principalmente pelo crescimento e desenvolvimento acelerados, fato que aumenta as necessidades nutricionais das crianças. Além disso, é uma época da vida em que são consolidados os hábitos alimentares. Assim, sabendo-se dos resultados positivos verificados na presente pesquisa, pode-se afirmar que os alunos foram estimulados a aumentar a prática regular de exercícios, bem como a se alimentar de uma forma mais saudável, o que poderá reduzir o risco de futuras doenças.

Destaca-se que menores percentuais de acertos para os conhecimentos em nutrição foram observados para os alimentos fontes de carboidratos (38,48\% - pré-intervenção e 86,30\% - pós-intervenção) e de proteínas (45,96\% - pré-intervenção e 86,36\% - pós-intervenção). Em geral, as crianças descrevem os alimentos conforme seu consumo habitual e, muitas vezes, não têm a capacidade de discernir os nutrientes presentes em cada grupo alimentar informado na pirâmide de alimentos (YOKOTA et al., 2010). Assim, fatores como o tipo de preparo dos alimentos, montagem dos pratos, horários de alimentação, representações simbólicas, dentre outros, podem influenciar na escolha, bem como na categorização de alimentos pelo público infantil, nem sempre sendo classificados nutricionalmente de forma adequada (JOMORI et al., 2008).

Cabe ressaltar, porém, o alto percentual de acertos para o grupo de alimentos fonte de açúcares e/ou gordura no período pré- 
-intervenção (80,46\%). Esse fato pode caracterizar uma ingestão elevada desses produtos pelas crianças, o que foi confirmado por Riveira e Souza (2006), avaliando escolares do município de Planaltina, DF. Segundo Fiates et al. (2006), uma explicação para esses resultados é a influência das propagandas direcionadas ao público infantil. Isto, porque apresentam uma linguagem adequada que satisfaz o consumidor. Da mesma forma, busca-se atrair a atenção das crianças melhorando a aparência, textura, aroma e sabor dos alimentos, favorecendo a escolha dos produtos oferecidos, além de uma alimentação compulsiva, o que pode desencadear futuramente doenças crônicas não transmissíveis (BORGES et al., 2009).

Diante dos resultados observados na Tabela 3 para o tópico de nutrição, verifica-se a necessidade de intervenções educativas constantes para o público infantil, já que as proteínas contêm aminoácidos essenciais, os quais necessitam ser ingeridos pela alimentação visando ao crescimento adequado. Do mesmo modo, os carboidratos são indispensáveis como fonte energética, considerando que, em geral, as crianças apresentam um elevado gasto calórico (OLIVEIRA, J. et al., 2010; YOKOTA et al., 2010).

No tópico de atividade física, os infantes tiveram menores percentuais de acertos quando se avaliaram os problemas causados pela falta de atividade física diária, as doenças que podem ser evitadas e o que pode causar a falta de exercícios. Nesse aspecto, pode-se observar que as ações preventivas relacionadas à prática de atividade física, principalmente aquelas pautadas na ocorrência de possíveis doenças crônicas, ainda não são totalmente conhecidas e/ ou esclarecidas entre escolares (BORGES et al., 2009), fato que demonstra a importância de práticas educacionais permanentes relacionadas à saúde infantil.

Na Figura 1, são apresentados os percentuais de acertos das crianças por sexo, em relação aos temas avaliados. 

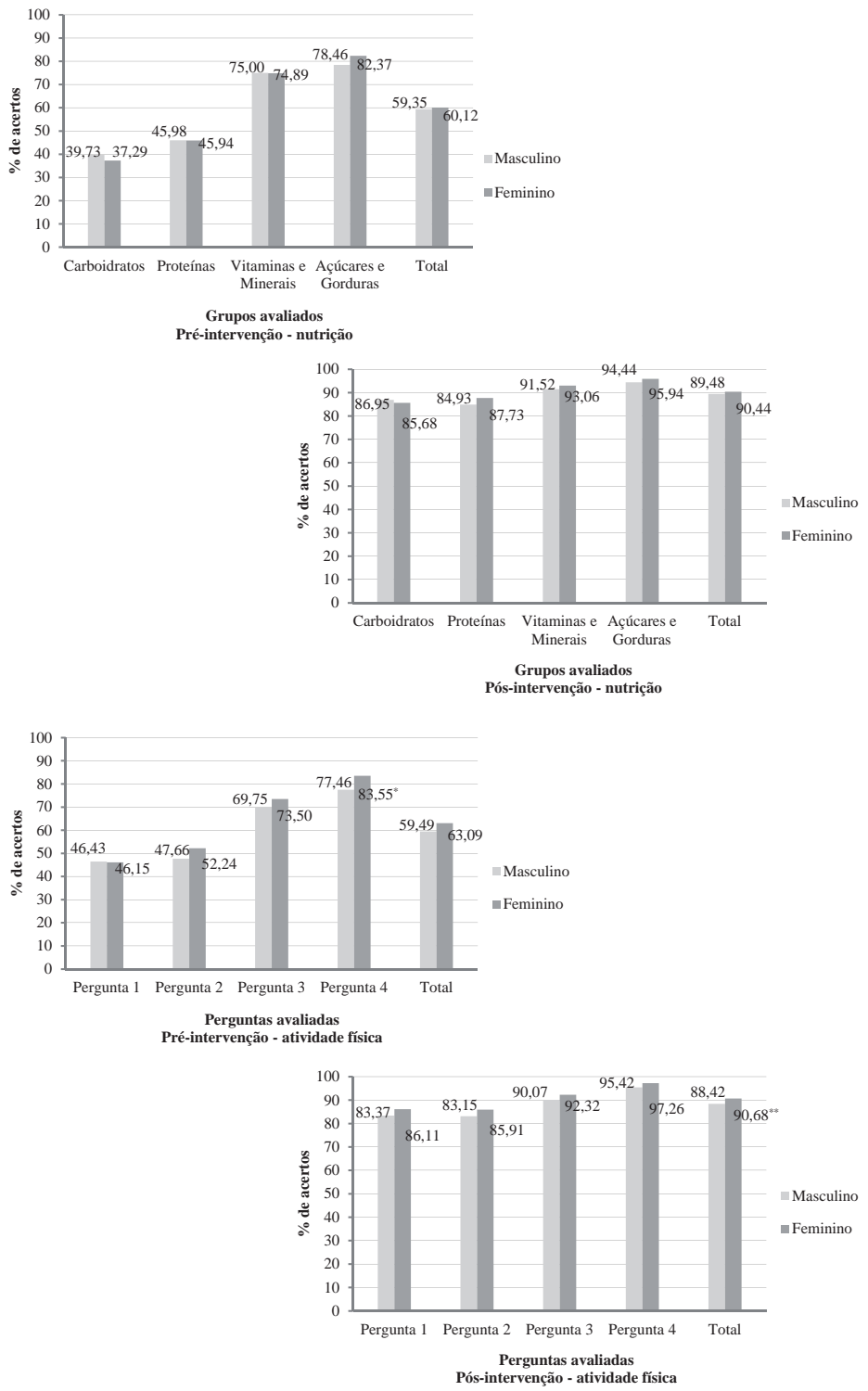

Nota: ${ }^{p} \mathrm{p}=0,018 ;{ }^{* *} \mathrm{p}=0,024$; análise pelo teste de t de student; $\mathrm{N}=458$.

Figura 1 - Percentuais de acertos entre os sexos em relação aos temas avaliados nos questionários sobre nutrição e atividade física, pré e pósintervenção. Guarapuava, PR, Brasil, 2015. 
Não se verificou diferença estatística $(p>0,05)$ entre os sexos para os acertos relacionados ao tema de nutrição, tanto no período pré quanto no pós-intervenção. Contudo, para o tópico de atividade física pré-intervenção (pergunta 4), houve maior percentual de acertos $(p=0,018)$ para o sexo feminino $(83,55 \%)$ comparado ao masculino (77,46\%). De forma similar, no período pós-intervenção (atividade física), as meninas tiveram maior percentual de acertos $(90,68 \%)$ que os meninos (88,42\%), quando se avaliou o total de respostas $(p=0,024)$, corroborando com Silveira e Silva (2011).

Segundo Medeiros et al. (2011), desde a infância existe uma cobrança maior da sociedade em relação ao sexo feminino, principalmente, nas questões de saúde e de aparência corporal (corpo mais magro). Assim, pode ocorrer uma procura precoce por conhecimentos relacionados à alimentação e atividade física pelas meninas, concordando com os resultados verificados na presente pesquisa. Contudo esse processo contínuo pode desencadear transtornos alimentares conhecidos como anorexia e bulimia (GONÇALVES et al., 2013).

\section{CONCLUSÃO}

A realização de intervenções educacionais como palestras explicativas, brincadeiras e dinâmicas com participação dos escolares, mostraram-se adequadas para melhorar os conhecimentos sobre nutrição e atividade física nesse público. Ressalta-se que a utilização de métodos lúdicos de aprendizagem permite um maior envolvimento e compreensão para o incentivo a um consumo alimentar saudável e a prática de atividade física pelas crianças.

As ações educativas no ambiente escolar promovem um maior envolvimento das crianças, favorecendo a realização das atividades em um ambiente mais agradável, considerando-se as condições existentes no local, intensificando, assim, a utilização de recursos humanos e didá- 
ticos. Nesse contexto, políticas públicas que visem a melhores condições de saúde à população devem ser incentivadas nas escolas e em outros locais, sempre por meio de constantes interações e diálogos entre os envolvidos nesse processo, com intuito de obter a construção do conhecimento, bem como mudanças e manutenção de hábitos saudáveis.

\section{AGRADECIMENTOS}

Os autores agradecem à Fundação Araucária de Apoio ao Desenvolvimento Científico e Tecnológico do Estado do Paraná pelo apoio no desenvolvimento da pesquisa.

\section{REFERÊNCIAS}

ALBUQUERQUE, S. A educação física na educação infantil. Curitiba: Expoente, 2008.

BERNART, A.; ZANARDO, V. P. S. Educação nutricional para crianças em escolas públicas de Erechim/RS. Vivências, Erechim, v. 7, n. 13, p. 71-79, out. 2011.

BERTIN, R. L.; MALKOWSKI J.; ZUTTER L. C. I.; ULBRICH A. Z. Estado nutricional, hábitos alimentares e conhecimentos de nutrição em escolares. Revista Paulista de Pediatria, São Paulo, v. 28, n. 3, p. 302-208, set. 2010.

BORGES, T. T.; ROMBALDI, A. J.; KNUTH, A. G.; HALLAL, P. C. Conhecimento sobre fatores de risco para doenças crônicas: estudo de base populacional. Cadernos de Saúde Pública, Rio de Janeiro, v. 25, n. 7, p. 1511-1520, jul. 2009.

BRASIL. Ministério da Saúde. Guia Alimentar para a população brasileira promovendo a alimentação saudável. Brasília: Ministério da Saúde, 2006. (Série A, Normas e Manuais Técnicos).

BRASIL. Secretaria de Atenção à Saúde. Orientações para a coleta e análise de dados antropométricos em serviços de saúde - Sistema de Vigilância Alimentar e Nutricional (SISVAN). Brasília: Ministério da Saúde, 2008.

CARMO, N.; GRINGER, C.; NETO, J. B. S.; FRANÇA, J. C.; VICTORINO, R.; PEREIRA, C. C. D. A. A importância da educação física escolar sobre aspectos de saúde: sedentarismo. Revista Educare CEUNSP, v. 1, n. 1, p. 21-29, 2013. 
Eficácia de intervenções educacionais sobre os conhecimentos em nutrição e atividade física de escolares

COSCRATO, G.; PINA, J. C.; MELLO, D. F. Utilização de atividades lúdicas na educação em saúde: uma revisão integrativa da literatura. Revista Acta Paulista de Enfermagem, São Paulo v. 23, n. 2, p. 257-263, mar./abr. 2010.

DEPARTMENT OF HEALTH AND HUMAN SERVICES (DHHS). Physical Activity Guidelines for Americans. Washington, USA: Department of Health and Human Services, 2008.

DOMingueS, M. R.; ARAÚJO, C. L. P.; GIGANTE, D. P. Conhecimento e percepção sobre exercício físico em uma população adulta urbana do sul do Brasil. Cadernos de Saúde Pública, Rio de Janeiro, v. 20, n. 1, p. 204-215, jan./fev. 2004.

ENES, C. C.; SLATER, B. Obesidade na adolescência e seus principais fatores determinantes. Revista Brasileira de Epidemiologia, São Paulo, v. 13, n. 1, p. 163-171, mar. 2010.

FARIAS, E. S.; CARVALHO, W. R. G.; GONÇALVES, E. M.; JÚNIOR, G. G. Efeito da atividade física programada sobre a aptidão física em escolares adolescentes. Revista Brasileira de Cineantropometria e Desempenho Humano, Santa Catarina, v. 12, n. 2, p. 98-105, 2010.

FERREIRA, S. H. Z.; BARREARA, S. D. Ambiente familiar e aprendizagem escolar em alunos da educação infantil. Revista Psico, Porto Alegre, v. 41, n. 4, p. 462-472, out./dez. 2010.

FIATES, G. R. M.; AMBONI, R. D. M. C.; TEIXEIRA, E. Marketing, hábitos alimentares e estado nutricional: aspectos polêmicos quando o tema é o consumidor infantil. Alimentos e Nutrição, Araraquara, v. 17, n. 1, p. 105112, jan./mar. 2006.

FREITAS, A. S. S.; COELHO, S. C.; RIBEIRO, R. L. Obesidade infantil: influência de hábitos alimentares inadequados. Revista Saúde \& Ambiente, Duque de Caxias, v. 4, n. 2, p. 9-14, jul./dez. 2009.

FREITAS, K. L. S. Envolver: ferramentas pedagógicas e educação nutricional. 2009. 63f. Trabalho de Conclusão de Curso (Curso de Nutrição) - Universidade Federal do Rio Grande do Norte, Natal, RN, 2009.

FRIEDRICH, R. R.; SCHUCH, I.; WAGNER, M. B. Effect or interventions on the body mass index of school-age studentes. Revista de Saúde Pública, São Paulo, v. 46, n. 3, p. 551-560 jun. 2012. 
GONÇALVES, A. J.; MOREIRA, E. A. M.; TRINDADE, E. B. S. M.; FIATES, G. M. R. Transtornos alimentares na infância e na adolescência. Revista Paulista de Pediatria, São Paulo, v. 31, n. 1, p. 96-103, jan./mar. 2013.

GONÇALVES, M. C. Repensando a Educação Física: da Educação Infantil ao Ensino Fundamental. Curitiba: Bolsa Nacional do Livro, 2007.

INSTITUTO BRASILEIRO DE GEOGRAFIA E ESTATÍSTICA (IBGE). Pesquisa de orçamentos familiares 2008-2009. Rio de Janeiro: IBGE, 2010.

JOMORI, M. K.; PROENÇA, R. P. C.; CALVO, C. M. Determinantes de escolha alimentar. Nutrição, Campinas, v. 21, n. 1, p. 63-73, jan./fev. 2008.

MEDEIROS, M. C. C.; CARDOSO, M. A. A.; PEREIRA, R. A. R.; ALVES, G .T. A.; FRANÇA, I. S. X.; COURA, A. S.; CARVALHO, D. F. Nutritional status and habits of life in school children. Revista Brasileira de Crescimento e Desenvolvimento Humano, São Paulo, v. 21, n. 3, p. 789-797, 2011.

MELLO, A. D. M.; MARCON, S. S.; HULSMEYER, A. P. C. R.; CATTAR, G. B. P.; AYRES, C. S. L. S.; SANTANA, R. G. Prevalência de sobrepeso e obesidade em crianças de seis a dez anos de escolas municipais da área urbana. Revista Paulista de Pediatria, São Paulo, v. 28, n. 1, p. 48-54, mar. 2010.

OLIVEIRA, J. C.; COSTA, S. D.; ROCHA, S. M. B. Educação nutricional com a atividade lúdica para escolares da rede municipal de ensino de Curitiba. Cadernos da Escola de Saúde, Curitiba, v. 1, n. 9, p. 100-116, 2011.

OLIVEIRA, J. R. P.; DIAS, A. S.; BOSCO, A. D.; ANGELICH, A. G.; ULSEFER, S.; MAGALHÃES, R. C.; SANTOS, S. Avaliação da capacidade respiratória, obesidade e capacidade física em crianças de 8 a 11 anos de idade. Revista Brasileira de Ciência e Movimento, Taguatinga, DF, v. 12, n. 23, p. 7-18, 2010.

OLIVEIRA, N. A.; FIETZ, V. R.; CARVALHO, E. A. I. Educação nutricional entre crianças. Anais do SEMEX. 8 SEMEX, Campo Grande, MS, n. 3, 2010.

PHILIPPI, S. T. Pirâmide dos alimentos: fundamentos básicos da Nutrição. São Paulo: Manole, 2014.

PONTES, T. E.; COSTA, T. F.; MARUM, A. B. R. F.; BRASIL, A. L. D.; TADDEI, J. A. A. C. Orientação nutricional de crianças e adolescentes e os novos padrões de consumo: propagandas, embalagens e rótulos. Revista Paulista de Pediatria, São Paulo, v. 27, n. 1, p. 99-105, mar. 2009. 
Eficácia de intervenções educacionais sobre os conhecimentos em nutrição e atividade física de escolares

PRADO, B. G. Consumo alimentar de escolares antes e após ações de educação nutricional, em Cuiabá-MT. 2011. 122 f. Dissertação (Pós-Graduação em Saúde Coletiva)- Universidade Federal do Mato Grosso, Instituto de Saúde Coletiva, Cuiabá, MT, 2011.

PRAZERES FILHO, A.; MACHADO, D. C. B.; GUERRA, R. R. R.; MARTINS, C. O.; GOMES, F. N.; GONÇALVES, F. J. Atividades físicas para criança. Música. [s.d.]. Disponível em: <http://lepafsdef.yolasite.com/resources/musica_criancas. pdf>. Acesso em: 4 mar. 2016.

PUBLIC HEALTH AGENCY OF CANADA (PHAC). Gotta move! Magazine for children 6-9 years of age. Canada's physical activity guide to healthy active living. Ottawa: PHAC, 2002.

RIVEIRA, F. S. R.; SOUZA, E. M. T. Consumo alimentar de escolares de uma comunidade rural. Comunicação em Ciências da Saúde, Samambaia, DF, v. 17, n. 2, p. 111-119, set. 2006.

SILVA, K. S.; NAHAS, M. V.; PERES, K. G.; LOPES, A. S. Fatores associados à atividade física, comportamento sedentário e participação na Educação Física em estudantes do Ensino Médio em Santa Catarina, Brasil. Cadernos de Saúde Pública, Rio de Janeiro, v. 2, n. 10, p. 2187-2200, out. 2009.

SILVA, P. V. C.; COSTA, A. L. J. Efeitos da atividade física para a saúde de crianças e adolescentes. Psicologia Argumento, Curitiba, v. 29, n. 64, p. 4150, jan./mar. 2011.

SILVEIRA, E. F.; SILVA, M. C. Conhecimentos sobre atividade física em estudantes de uma cidade do sul do Brasil. Revista Motriz, Rio Claro, v. 17, n. 3, p. 456-467, jul./set. 2011.

SIMON, V. G. N.; SOUZA, J. M. P.; LEONE, C.; SOUZA, S. B. Prevalência de sobrepeso e obesidade em crianças de dois a seis anos matriculadas em escolas particulares no município de São Paulo. Revista Brasileira de Crescimento e Desenvolvimento Humano, São Paulo, v. 19, n. 2, p. 211-218, 2009.

SIQUEIRA, P. P.; ALVES, J. G. B.; FIGUEIROA, J. N. Fatores associados ao excesso de peso em crianças de uma favela do Nordeste brasileiro. Revista Paulista de Pediatria, São Paulo, v. 27, n. 3, p. 251-257, set. 2009.

SOCIEDADE BRASILEIRA DE PEDIATRIA (SBP). Atividade física na infância e na adolescência: guia prático para o pediatra. Rio de Janeiro: SBP, 2008. 
YOKOTA, R. T. C.; VASCONCELOS, T. F.; PINHEIRO, A. R. O.; SCHMITZ, B. A. S.; COITINHO, D. C, RODRIGUES, M. L. C. F. Projeto "a escola promovendo hábitos alimentares saudáveis": comparação de duas estratégias de educação nutricional no Distrito Federal, Brasil. Revista de Nutrição, Campinas, v. 23, n. 1, p.37-47, jan./fev. 2010. 